\title{
AVALIAÇÃO DO PERFIL SENSORIAL DE AMÊNDOAS DE CUPUAÇU E CACAU TORRADAS UTILIZANDO ANÁLISE DESCRITIVA QUANTITATIVA
}

\author{
MARISE BONIFÁCIO QUEIROZ * \\ NELSON HORÁCIO PEZOA GARCIA **
}

\begin{abstract}
Utilizou-se a análise descritiva quantitativa (ADQ) para desenvolver terminologia descritiva e perfil sensorial de amêndoas de cupuaçu e cacau torradas. Quatro amostras de cupuaçu torradas em forno elétrico rotativo a $150 \stackrel{\circ}{\mathrm{C}}$ por $38,40,42$ e 44 minutos e uma amostra de cacau torrada por 38 minutos foram avaliadas sensorialmente $e$ comparadas entre si. Foram gerados 12 termos que melhor descreveram as similaridades e diferenças entre as amostras, sendo apontados $8 \mathrm{com}$ maior predominância segundo a análise de componente principal (ACP). Equipe de 9 julgadores foi selecionada com base no poder de discriminação $\left(F_{\text {amostra }}\right.$ significativo para $\left.p \leq 0,50\right)$, repetibilidade

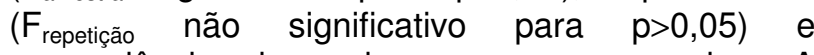
concordância de cada um com a equipe. A intensidade de cada descritor ou atributo foi fixada em escala não estruturada de $10 \mathrm{~cm}$, ancorada por extremos que variavam de fraco para forte, baixa para alta, etc. Os dados obtidos foram avaliados estatisticamente (ANOVA, Teste de Tukey e ACP). Observou-se que o cacau torrado apresentou características sensoriais diferentes do cupuaçu, sendo bem mais ácido e amargo, com coloração mais escura e acentuado sabor de chocolate. Já o cupuaçu apresentou sabor de chocolate mais suave e mais doce que o cacau.
\end{abstract}

* Mestre em Tecnologia de Alimentos pela Universidade de Campinas (UNICAMP) e Pesquisadora do Instituto de Tecnologia de Alimentos (ITAL), CEREAL CHOCOTEC/ITAL, Campinas, SP. (e-mail: bqueiroz@ital.org.br).

** Prof. Dr., Departamento de Tecnologia de Alimentos, Faculdade de Engenharia de Alimentos (FEA), UNICAMP, Campinas, SP. 
O cupuaçu (Theobroma grandiflorum), um dos mais importantes frutos típicos da amazônia, é cultivado em todo o Estado do Pará, norte do Maranhão e Tocantins, havendo também cultivo de espécimes isoladas nos Estados de São Paulo, Bahia e Rio de Janeiro (CAVALCANTE, 1976; VENTURIERI et al.,1988). É uma cultura emergente, com potencial para conquistar novos mercados, pois destaca-se pelo aroma e sabor agradáveis aliados à diversidade de produtos e subprodutos oriundos do fruto (RIBEIRO, 1996).

Estudos mostram que as sementes de cupuaçu desenvolvem fino aroma característico, similar ao do chocolate, quando fermentadas e torradas. Apresentam na sua composição gordura de sabor suave, que se assemelha à manteiga de cacau, no que se refere a composição química e características sensoriais (COUTINHO, 1969, ARAGÃO, 1992, NAZARÉ, 1996, VENTURIERI, 1988).

Segundo NAZARÉ (1996) são inúmeros os produtos derivados do cupuaçu, processados muitas vezes de forma artesanal na região de cultivo. De sua polpa pode-se obter cremes, tortas, sorvetes, balas, bombons, geléias, licores, doces, compotas, polpa seca e granulada, etc. As sementes servem para fabricação de produtos semelhantes ao chocolate e ao cacau em pó, sendo a cor, o aroma e o sabor similares ao chocolate, porém com características muito peculiares.

A avaliação sensorial dos alimentos tem como objetivo oferecer subsídios que possam melhorar a qualidade dos produtos, verificar os efeitos do seu processamento ou armazenamento, caracterizar novos produtos em estudo e para efetuar pesquisas de mercado (AMERINE et al., 1965). Os métodos analíticos descritivos, enumeram as características percebidas e suas intensidades. A técnica inclui avaliação e comparação de sabores, discriminando-os seja pela descrição das impressões do produto como um todo, como por suas características individuais. Baseia-se no conceito de que uma pessoa pode ser treinada para perceber e reconhecer características sensoriais individuais de determinado produto, o grau de sua intensidade, e com treinamento apropriado alcançar concordância com os outros membros do grupo. Desta forma, pode-se substituir a avaliação de um expert individual por grupo de pessoas treinadas, que proporcionam resposta coletiva, a qual na maioria dos métodos descritivos pode ser analisada estatisticamente (SILVA, 1992).

A análise descritiva quantitativa (ADQ) avalia, através de pontos, a aparência, a cor, o odor, o sabor e a textura de determinado produto, de acordo com a ordem em que são detectadas pelos provadores. Resulta em completa descrição sensorial do produto em teste e fornece base para determinar as características que são importantes para sua aceitação, bem 
como para auxiliar a identificação dos seus ingredientes, variáveis de processo, etc. (STONE et al., 1985).

O presente estudo teve por objetivo avaliar, com base na análise descritiva quantitativa, as propriedades sensoriais de amêndoas de cupuaçu torradas em diferentes tempos e compará-las sensorialmente com amêndoas de cacau, torradas em tempo favorável ao desenvolvimento de sabor de chocolate, conforme estabelecido em estudo anterior. Avaliou-se também a possibilidade de utilização do cupuaçu como matéria-prima na elaboração de produtos similares ao chocolate, coberturas e congêneres, aproveitando material que vem sendo subutilizado.

\section{MATERIAL E MÉTODOS}

As amostras de amêndoas de cupuaçu e cacau, torradas em forno elétrico rotativo, em diferentes tempos, a $150^{\circ} \mathrm{C}$, utilizadas na avaliação sensorial foram as seguintes:

CA 38: nibs* de cacau torrado por 38 minutos

CP 38: amêndoa de cupuaçu torrada por 38 minutos

CP 40: amêndoa de cupuaçu torrada por 40 minutos

CP 42: amêndoa de cupuaçu torrada por 42 minutos

CP 44: amêndoa de cupuaçu torrada por 44 minutos

*fragmentos do cotilédone da amêndoa de cacau com tamanhos compreendidos entre 4-6mm.

As amostras foram preparadas suspendendo-se o liquor de cupuaçu e cacau em água fervente, em concentração de $5 \%$, as quais foram servidas aos provadores a $50 \stackrel{\circ}{\circ}$, em copos plásticos de $50 \mathrm{~mL}$, codificados com três dígitos.

\subsection{ANÁLISE SENSORIAL}

A análise descritiva quantitativa (ADQ) compreendeu as etapas de desenvolvimento de terminologia descritiva, treinamento e seleção dos provadores, retreinamento da equipe e avaliação das amostras.

\subsubsection{Desenvolvimento de terminologia descritiva}

Aplicou-se o método Grid (MOSKOWITZ, 1983) para as amostras avaliadas a fim de se obter a lista de descritores. Foram apresentadas duas amostras de cada vez para 20 provadores, os quais anotaram as similaridades e diferenças entre elas, quanto aos atributos de aparência, aroma e sabor. Realizou-se posteriormente discussões abertas com os 
provadores para a elaboração da lista dos descritores mais importantes, eliminando-se termos sinônimos, duvidosos ou redundantes e ainda aqueles que apareceram com baixa freqüência. Esta fase iniciou-se com 20 provadores e ao final somente 15 puderam participar efetivamente do treinamento.

Para cada atributo selecionado em concordância com a equipe, quando possível foi apresentado material de referência, bem como referências de extremos de escala para padronizar o uso de cada termo descritivo, pelos provadores.

A intensidade de cada descritor ou atributo foi fixada em escala não estruturada de $10 \mathrm{~cm}$, ancorada por extremos que variavam de fraco para forte, baixa para alta, nenhuma para alta e assim por diante, com os quais foi possível a elaboração da ficha de avaliação para as amostras.

\subsubsection{Treinamento e seleção dos provadores}

Reuniu-se a equipe de provadores em sessões de treinamento, nas quais foram apresentadas amostras de referências, com seus respectivos extremos de escala para cada um dos atributos selecionados, de modo que pudessem atribuir valores na mesma região da escala de avaliação.

Para a etapa de seleção dos provadores as amostras foram servidas de acordo com delineamento de blocos completos casualizados, sendo que cada provador experimentou 5 amostras com 3 repetições, utilizando-se ficha de avaliação com os extremos de escala para os atributos selecionados. Desta forma, foi possível selecionar os provadores com maior poder de discriminação, repetibilidade e concordância com a equipe, mediante análises estatísticas. Utilizou-se a análise de variância (ANOVA) do pacote estatístico SAS (1989) para cada provador e atributo, considerando-se as causas de variação amostra e repetição. Obteve-se a probabilidade de $F_{\text {amostra }}$ e a probabilidade de $F_{\text {repetição, que foram utilizadas }}$ na seleção dos provadores com base em seu poder de discriminação $\left(F_{\text {amostra }}\right.$ significativo para $\left.p \leq 0,50\right)$ e repetibilidade $\left(F_{\text {repetição não significativo }}\right.$ para $p>0,05$ ) entre as amostras. Aplicou-se o teste de Tukey para obter a comparação entre as médias da equipe por atributo, as quais foram comparadas com as médias de cada provador e cada atributo, visando selecionar os provadores que concordavam com a equipe.

\subsubsection{Retreinamento da equipe e avaliação das amostras}

Após a seleção dos provadores efetuou-se novo treinamento com aqueles que, mesmo selecionados, ainda apresentavam certo desvio. Utilizou-se para tal a avaliação dos extremos de escala para os atributos com maior dificuldade de percepção por estes provadores, em sessões individuais. 
Após este treinamento as amostras foram servidas de acordo com a apresentação de blocos completos casualizados, para novamente os provadores avaliarem 5 amostras com três repetições. As amostras foram servidas em copos plásticos descartáveis de $50 \mathrm{~mL}$, codificados com três dígitos, na temperatura de 50 드. mantendo-se os padrões e referências de extremos de escala à disposição dos provadores durante todas as sessões de avaliação.

\subsubsection{Análise dos resultados}

Utilizou-se a ANOVA de dois fatores (amostra e provador) e também aplicou-se o teste de Tukey para comparação das médias das amostras. $O$ pacote estatístico SAS (1989) serviu ainda para a análise de componentes principais $(\mathrm{ACP})$.

\subsection{TESTE DE ACEITAÇÃO}

Com a amostra de cupuaçu torrada, que obteve os melhores resultados para sabor e aroma de chocolate, elaborou-se produto similar ao chocolate, formulado com $55 \%$ de liquor de cupuaçu e $45 \%$ de açúcar.

Realizou-se o refino da mistura em moinho de três cilindros, com o mínimo espaçamento possível entre os cilindros até a obtenção de massa refinada. Para a simulação da conchagem, utilizou-se batedeira planetária acoplada em banho-maria, com temperatura média de $60^{\circ} \mathrm{C}$, no qual a massa permaneceu por aproximadamente 8 horas, sob constante agitação.

A massa conchada foi temperada em mesa de mármore até temperatura

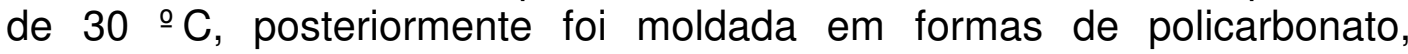
acondicionada em geladeira para 0 endurecimento e finalmente desmoldada. O produto obtido foi submetido à análise sensorial de aceitação, utilizando-se ficha com escala hedônica estruturada de 9 pontos e 40 julgadores não treinados.

\section{RESULTADOS E DISCUSSÃO}

\subsection{DESENVOLVIMENTO DE TERMINOLOGIA DESCRITIVA}

Aplicando-se o Método GRID obteve-se lista inicial de 45 termos descritores entre atributos de aparência, aroma e sabor. Em discussões abertas realizadas com os provadores eliminaram-se termos sinônimos, duvidosos ou redundantes e aqueles que apareceram com freqüência muito baixa. Desta forma, chegou-se a nova lista com 12 termos que melhor descreveram as amostras analisadas (Tabela 1). 


\section{TABELA 1 - DEFINICÕES DE DESCRITORES E PADRÕES DE REFERẾNCIA USADOS DURANTE A AVALIAÇÃOO DE AMÊNDOAS DE CUPUAÇU E CACAU TORRADAS}

\begin{tabular}{|c|c|}
\hline DESCRITORES & DEFINIÇÃO E REFERÊNCIAS \\
\hline $\begin{array}{l}\text { Cor marrom } \\
(\mathrm{COR})\end{array}$ & $\begin{array}{l}\text { Cor marrom característica de chocolate. } \\
\text { Clara: } 100 \mathrm{~mL} \text { de solução } 5 \% \text { de cupuaçu cru. } \\
\text { Escura: } 100 \mathrm{~mL} \text { de solução5\% de cacau torrado. }\end{array}$ \\
\hline $\begin{array}{l}\text { Aparência gordurosa } \\
\text { (GOR) }\end{array}$ & $\begin{array}{l}\text { Visualização de partículas de gordura em suspensão na superfície. } \\
\text { Pouco: } 100 \mathrm{~mL} \text { de solução } 5 \% \text { de cupиaçu cru. } \\
\text { Muito: } 100 \mathrm{~mL} \text { de solução } 5 \% \text { de cupuaçu mais } 3 \text { gotas de óleo de } \\
\text { soja. }\end{array}$ \\
\hline $\begin{array}{l}\text { Aparência encorpada } \\
\text { (ENC) }\end{array}$ & $\begin{array}{l}\text { Atributo associado à corpo. } \\
\text { Pouco: } 100 \mathrm{~mL} \text { de solução } 3 \% \text { de cupuaçu. } \\
\text { Muito: } 100 \mathrm{~mL} \text { de solução } 10 \% \text { de cupuaçu. }\end{array}$ \\
\hline $\begin{array}{l}\text { Aroma chocolate } \\
(\mathrm{ACHO})\end{array}$ & $\begin{array}{l}\text { Aroma característico de chocolate. } \\
\text { Fraco: } 100 \mathrm{~mL} \text { de solução } 5 \% \text { de cupuaçu cru. } \\
\text { Forte: } 100 \mathrm{~mL} \text { de solução } 5 \% \text { de cacau torrado mais } 5 \mathrm{~g} \text { de } \\
\text { chocolate em pó comercial. }\end{array}$ \\
\hline $\begin{array}{l}\text { Aroma ácido } \\
\text { (AACI) }\end{array}$ & $\begin{array}{l}\text { Atributo associado à ácido acético produzido durante a fermentação. } \\
\text { Fraco: } 100 \mathrm{~mL} \text { de solução } 5 \% \text { de cupuaçu torrado } 50 \mathrm{~min} / 150^{\circ} \mathrm{C} \text {. } \\
\text { Forte: } 100 \mathrm{~mL} \text { de solução } 5 \% \text { de cupuaçu cru, adicionado de } 1,0 \mathrm{~mL} \\
\text { de ácido acético. }\end{array}$ \\
\hline $\begin{array}{l}\text { Aroma doce } \\
\text { (ADOC) }\end{array}$ & $\begin{array}{l}\text { Aroma característico adocicado, associado à calda de açúcar. } \\
\text { Fraco: calda de sacarose a } 10^{\circ} \text { Brix. } \\
\text { Forte: calda de sacarose a } 40^{\circ} \text { Brix. }\end{array}$ \\
\hline $\begin{array}{l}\text { Aroma torrado } \\
\text { (ATOR) }\end{array}$ & $\begin{array}{l}\text { Aroma característico de produto que sofreu torração. } \\
\text { Fraco: solução } 5 \% \text { de cupuaçu cru. } \\
\text { Forte: solução } 5 \% \text { de cupuaçu torrado } 50 \mathrm{~min} / 150{ }^{\circ} \mathrm{C} \text {. }\end{array}$ \\
\hline $\begin{array}{l}\text { Sabor torrado } \\
\text { (STOR) }\end{array}$ & $\begin{array}{l}\text { Sabor característico de produto torrado. } \\
\text { Fraco: solução } 5 \% \text { de cupuaçu cru. } \\
\text { Forte: solução } 5 \% \text { de cupuaçu torrado } 50 \mathrm{~min} / 150^{\circ} \mathrm{C} \text {. }\end{array}$ \\
\hline $\begin{array}{l}\text { Sabor chocolate } \\
(\mathrm{SCHO})\end{array}$ & $\begin{array}{l}\text { Sabor residual de chocolate amargo (sem açúcar). } \\
\text { Fraco: solução } 5 \% \text { de cupuaçu cru. } \\
\text { Forte: } 100 \text { mL de solução } 5 \% \text { de cacau torrado por empresa mais } \\
5 \text { g de cacau em pó sem açúcar. }\end{array}$ \\
\hline $\begin{array}{l}\text { Sabor ácido } \\
\text { (SACI) }\end{array}$ & $\begin{array}{l}\text { Sabor associado à ácido acético, produzido durante a fermentação. } \\
\text { Fraco: solução } 5 \% \text { de cupиaçu torrado } 50 \mathrm{~min} / 150{ }^{\circ} \mathrm{C} \text {. } \\
\text { Forte: solução } 5 \% \text { de cupuaçu cru mais } 0,13 \mathrm{~g} \text { de ácido cítrico. }\end{array}$ \\
\hline $\begin{array}{l}\text { Sabor amargo } \\
\text { (SAMA) }\end{array}$ & $\begin{array}{l}\text { Sabor residual marcante característico de cacau. } \\
\text { Fraco: solução } 5 \% \text { de cupuaçu torrado } 25 \mathrm{~min} / 150^{\circ} \mathrm{C} \text {. } \\
\text { Forte: liquor puro de cacau torrado por empresa. }\end{array}$ \\
\hline $\begin{array}{l}\text { Adstringência } \\
\text { (SADS) }\end{array}$ & $\begin{array}{l}\text { Sensação que "amarra na boca". } \\
\text { Fraco: banana madura. } \\
\text { Forte: banana verde. }\end{array}$ \\
\hline
\end{tabular}


Para cada atributo selecionado foi possível apresentar padrões de referência, representando os extremos da escala, bem como definições para esclarecer aos provadores o significado daquele atributo que estava sendo avaliado (Tabela 1).

Os termos desenvolvidos foram utilizados para compor a ficha de avaliação das amostras, com escala não estruturada de 10 pontos.

\subsection{SELEÇÃO DOS PROVADORES}

Com base no poder de discriminação $\left(F_{\text {amostra }}\right)$, repetibilidade $\left(F_{\text {repetição }}\right)$ e concordância com a equipe foi possível selecionar 9 dos 15 provadores que compunham o grupo. No caso da probabilidade de $F_{\text {amostra }}$ (Tabela 2), os provadores 7 e 10 atribuíram valores maiores que 0,5 para 5 atributos, o que indicou poder de discriminação não muito satisfatório. Outros provadores, como o 3, 9 e 13 também não tiveram resultados tão bons para probabilidade de $\mathrm{F}_{\text {amostra }}(3$ atributos com valores maiores que 0,5 ).

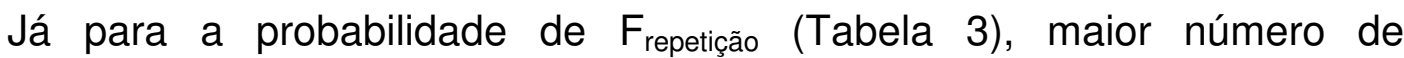
provadores atribuíram valores inferiores a 0,05 para pelo menos 3 atributos, o que caracteriza menor poder de repetibilidade entre as amostras, com destaque para os provadores 1, 4, 8, 9, 11, 12 e 14 .

Comparando-se os valores das médias da equipe com as médias de cada provador para os 12 atributos analisados (Tabela 4) pode-se observar que, os que mais discordaram foram o 2, 7, 8 e 9. A discordância dos provadores com relação à equipe foi caracterizada da seguinte maneira:

- provador distinguiu as amostras, porém atribuiu média diferente da equipe, sendo esta ora maior ora menor;

- provador distinguiu as amostras, mas inverteu os valores, atribuindo média mais alta para a amostra que a equipe atribuiu baixa e vice-versa;

- provador não percebeu a diferença entre as amostras quando a equipe percebeu.

Com base nos resultados decidiu-se eliminar os provadores 2, 7, 8, 9, 10 e 13. Os provadores que não foram eliminados, mas que ainda apresentaram problemas (discriminação, repetibilidade e concordância) passaram por sessões de retreinamento. Os provadores que compuseram a equipe selecionada para a análise descritiva apresentaram depois do retreinamento melhoria na discriminação e repetibilidade entre as amostras, indicando grau adequado de treinamento. 


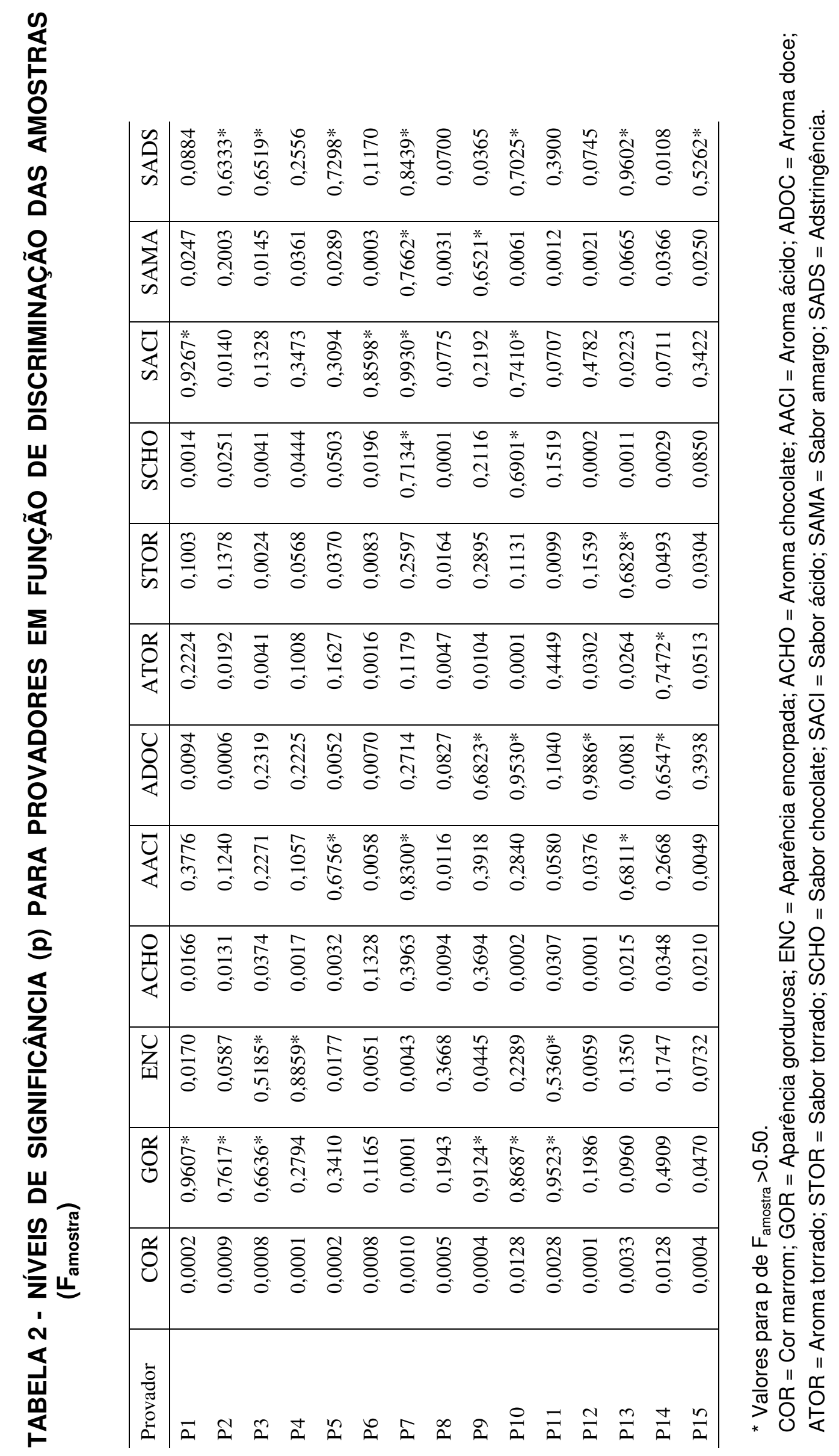




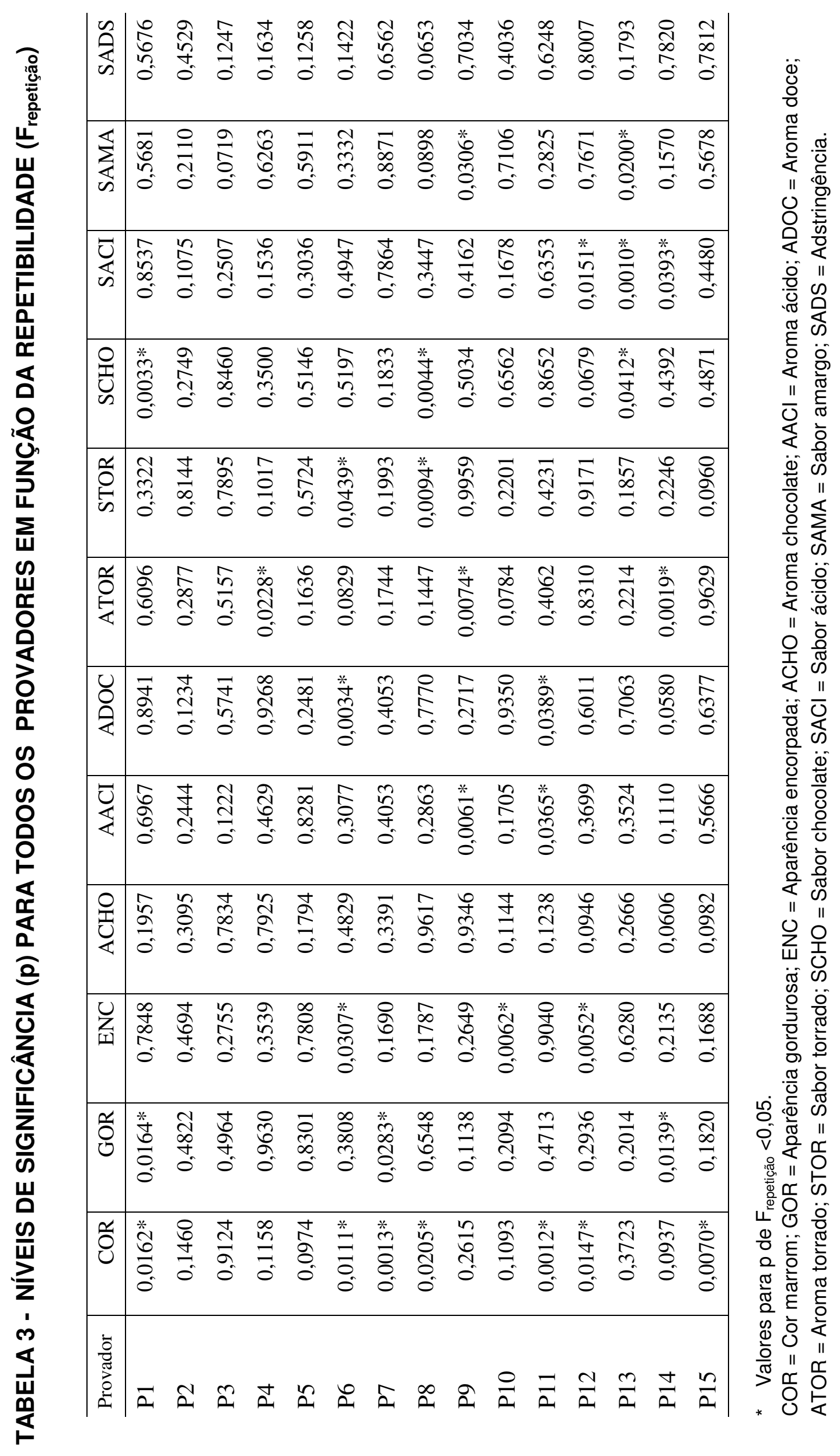




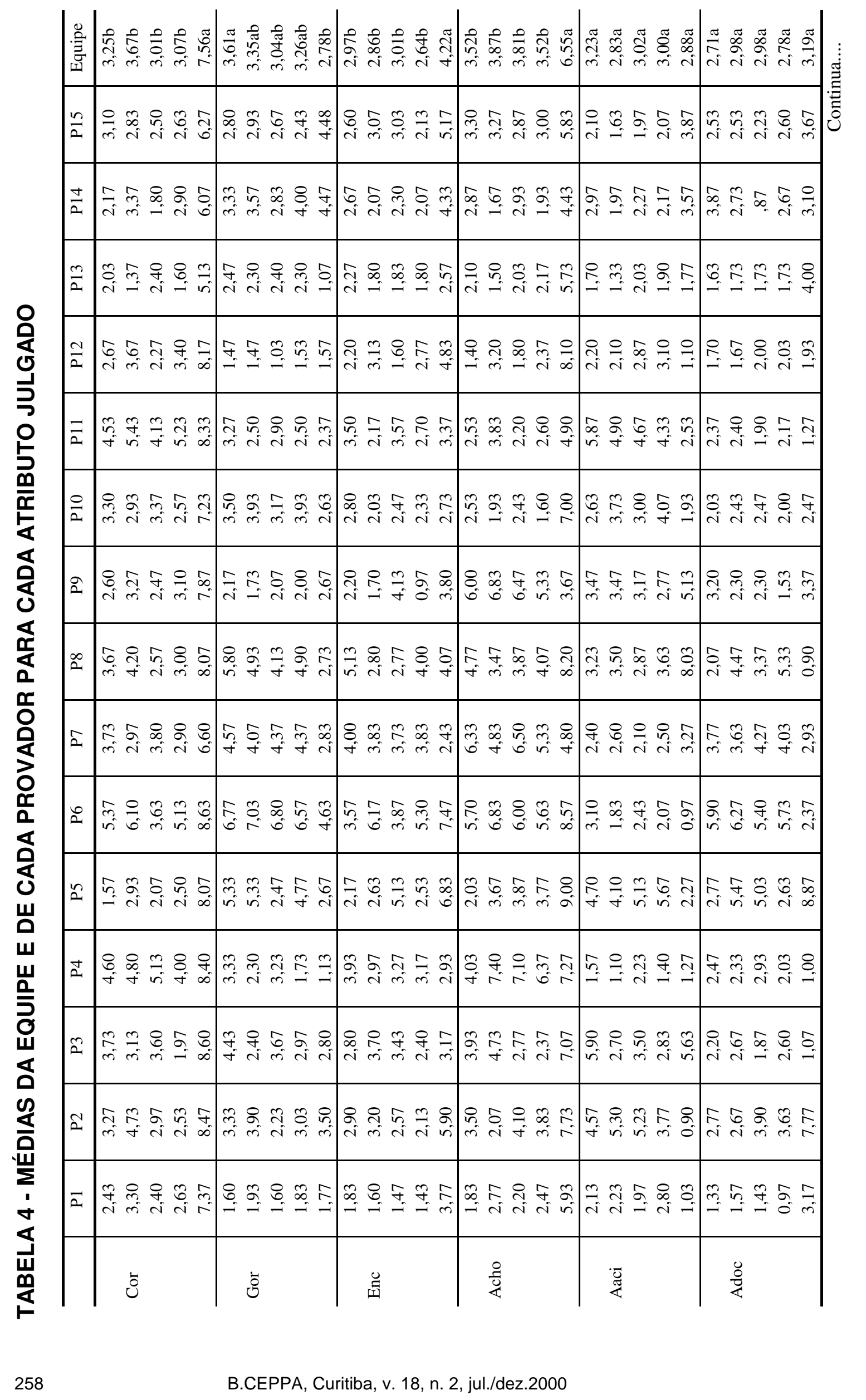




\begin{tabular}{|c|c|c|c|c|c|c|c|}
\hline 产 & 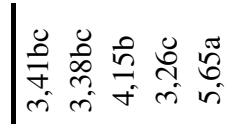 & 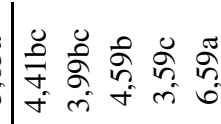 & 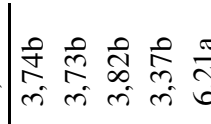 & 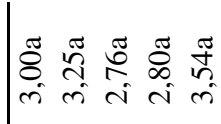 & 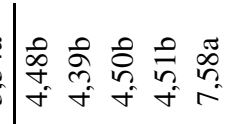 & 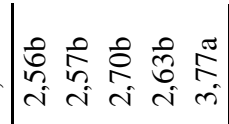 & 蒙 \\
\hline$\frac{n}{2}$ & 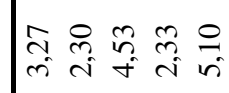 & 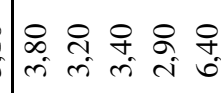 & 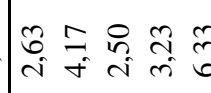 & $\mid \begin{array}{ccccc}0 & 0 & 0 & \tilde{n} & m \\
i & i & i & i & m\end{array}$ & 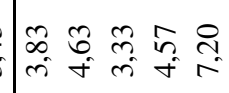 & 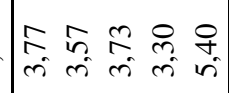 & 要 \\
\hline$\frac{\pi}{a}$ & 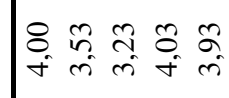 & 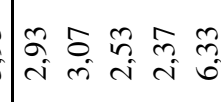 & 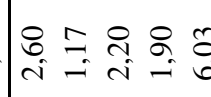 & 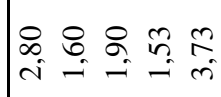 & 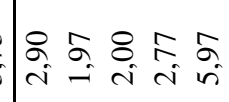 & 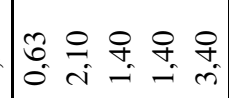 & (্) \\
\hline$\frac{m}{2}$ & 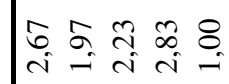 & 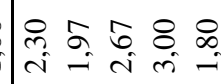 & 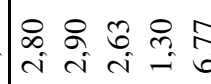 & 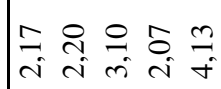 & 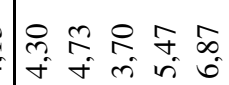 & 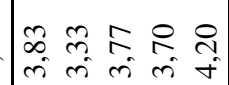 & II \\
\hline$\frac{2}{2}$ & 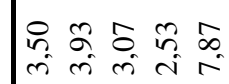 & 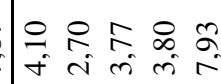 & 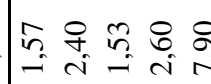 & 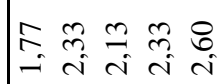 & 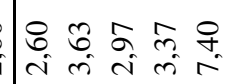 & 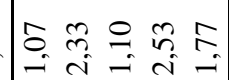 & \\
\hline $\bar{z}$ & 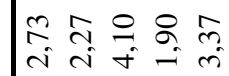 & 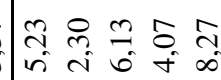 & 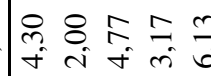 & $\begin{array}{lll}0 & 8 & 0 \\
0 & 0 & 0\end{array}$ & 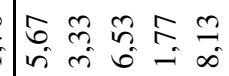 & nn & $\overline{0}$ \\
\hline$\frac{\circ}{2}$ & 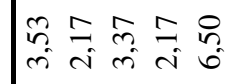 & 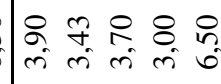 & 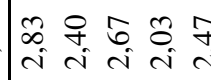 & 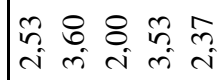 & 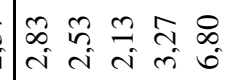 & m & II \\
\hline$\hat{\imath}$ & 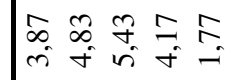 & $\begin{array}{l}\Rightarrow \\
n \\
n\end{array}$ & 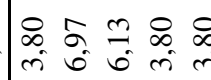 & 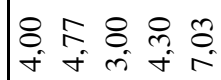 & $\begin{array}{l}m=0 \\
\therefore=0\end{array}$ & 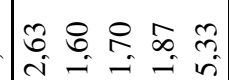 & 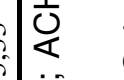 \\
\hline$\infty$ & 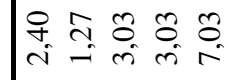 & 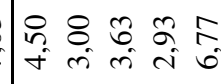 & 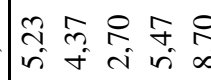 & 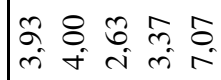 & 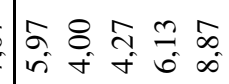 & 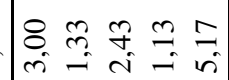 & 0 \\
\hline$\underline{\Omega}$ & 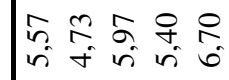 & 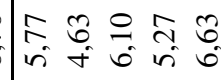 & 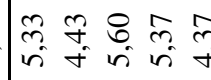 & 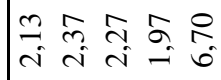 & 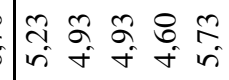 & 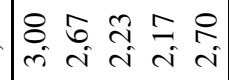 & i \\
\hline 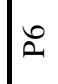 & 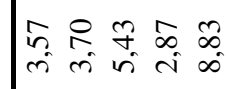 & 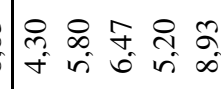 & 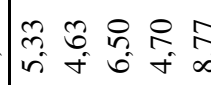 & 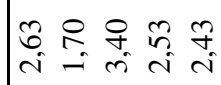 & 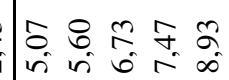 & 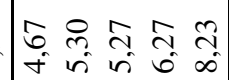 & 0 \\
\hline$\approx$ & $\begin{array}{lllll}0 & n & 0 & 0 & 0 \\
0 & m & 0 & 0 & 0\end{array}$ & 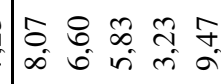 & 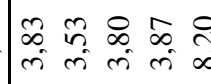 & 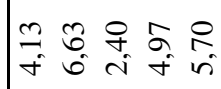 & 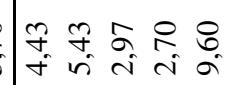 & 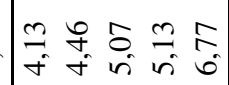 & ס \\
\hline I & 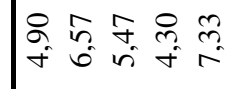 & 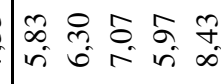 & 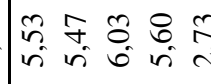 & 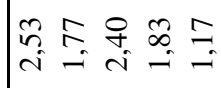 & 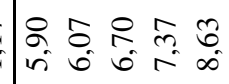 & {$\left[\begin{array}{ccc}\infty & m & m \\
-0 & 0 & 0\end{array}\right.$} & $\frac{\pi}{0}$ \\
\hline$\approx$ & 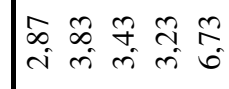 & 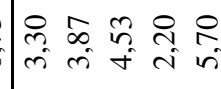 & 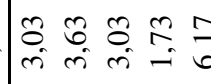 & 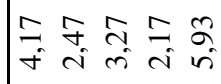 & 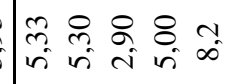 & 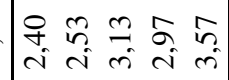 & 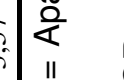 \\
\hline$\approx$ & 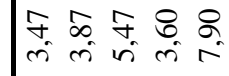 & 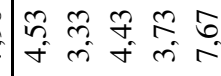 & 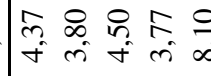 & 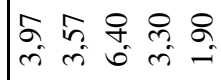 & 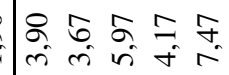 & 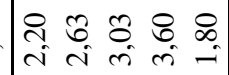 & \\
\hline $\bar{z}$ & 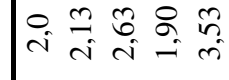 & 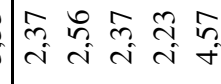 & 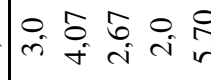 & 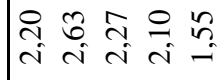 & 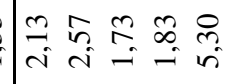 & 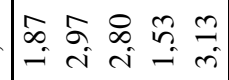 & \\
\hline & 芩 & 竞 & 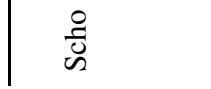 & $\overline{\mathscr{E}}$ & 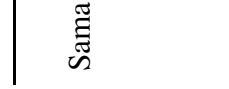 & 逽 & 10 \\
\hline
\end{tabular}




\subsection{ANÁLISE DESCRITIVA DAS AMOSTRAS DE AMÊNDOA DE CUPUAÇU E CACAU TORRADAS}

As médias das amostras comparadas pelo teste de Tukey estão apresentadas na Tabela 5. Valores marcados com a mesma letra, na mesma linha não diferem significativamente entre si para $p \leq 0,05$.

\section{TABELA 5 - MÉDIAS DAS AMOSTRAS POR ATRIBUTO AVALIADO}

\begin{tabular}{l|l|l|l|l|l}
\hline \multirow{2}{*}{ Atributo } & \multicolumn{5}{|c}{ AMOSTRAS } \\
\cline { 2 - 6 } & Cp38 & Cp40 & Cp42 & Cp44 & Ca38 \\
\hline COR & $3,05^{\mathrm{c}}$ & $3,37^{\mathrm{bc}}$ & $3,46^{\mathrm{bc}}$ & $3,78^{\mathrm{b}}$ & $7,66^{\mathrm{a}}$ \\
\hline GOR & $3,43^{\mathrm{a}}$ & $3,24^{\mathrm{a}}$ & $2,99^{\mathrm{a}}$ & $3,37^{\mathrm{a}}$ & $3,07^{\mathrm{a}}$ \\
\hline ENC & $2,60^{\mathrm{b}}$ & $3,02^{\mathrm{b}}$ & $3,15^{\mathrm{b}}$ & $2,98^{\mathrm{b}}$ & $4,80^{\mathrm{a}}$ \\
\hline ACHO & $3,18^{\mathrm{c}}$ & $4,37^{\mathrm{b}}$ & $4,34^{\mathrm{b}}$ & $3,35^{\mathrm{c}}$ & $6,79^{\mathrm{a}}$ \\
\hline AACI & $3,65^{\mathrm{a}}$ & $2,69^{\mathrm{b}}$ & $2,81^{\mathrm{b}}$ & $2,61^{\mathrm{b}}$ & $2,77^{\mathrm{b}}$ \\
\hline ADOC & $3,21^{\mathrm{a}}$ & $3,35^{\mathrm{a}}$ & $3,22^{\mathrm{a}}$ & $2,44^{\mathrm{b}}$ & $1,48^{\mathrm{c}}$ \\
\hline ATOR & $3,14^{\mathrm{d}}$ & $3,52^{\mathrm{cd}}$ & $4,15^{\mathrm{c}}$ & $4,65^{\mathrm{b}}$ & $6,07^{\mathrm{a}}$ \\
\hline STOR & $4,32^{\mathrm{b}}$ & $3,80^{\mathrm{b}}$ & $4,49^{\mathrm{b}}$ & $3,51^{\mathrm{b}}$ & $7,34^{\mathrm{a}}$ \\
\hline SCHO & $3,15^{\mathrm{b}}$ & $3,54^{\mathrm{b}}$ & $3,77^{\mathrm{b}}$ & $3,40^{\mathrm{b}}$ & $5,18^{\mathrm{a}}$ \\
\hline SACI & $4,00^{\mathrm{a}}$ & $3,26^{\mathrm{b}}$ & $2,06^{\mathrm{c}}$ & $2,35^{\mathrm{c}}$ & $2,97^{\mathrm{b}}$ \\
\hline SAMA & $4,21^{\mathrm{b}}$ & $4,28^{\mathrm{b}}$ & $3,98^{\mathrm{b}}$ & $4,09^{\mathrm{b}}$ & $7,70^{\mathrm{a}}$ \\
\hline SADS & $2,80^{\mathrm{b}}$ & $2,67^{\mathrm{b}}$ & $2,79^{\mathrm{b}}$ & $2,85^{\mathrm{b}}$ & $3,81^{\mathrm{a}}$ \\
\hline
\end{tabular}

$\mathrm{COR}=$ Cor marrom; GOR = Aparência gordurosa ENC = Aparência encorpada; $\mathrm{ACHO}=$ Aroma chocolate; $\mathrm{AACl}=$ Aroma ácido; $\mathrm{ADOC}=$ Aroma doce; ATOR = Aroma torrado; $\mathrm{STOR}=$ Sabor torrado; $\mathrm{SCHO}=$ Sabor chocolate; $\mathrm{SACl}=$ Sabor ácido; SAMA = Sabor amargo; SADS = Adstringência.

Os dados coletados foram submetidos a análise multivariada de componente principal (ACP), ilustrando-se na Figura 1 as propriedades sensoriais de cada amostra.

Para as amostras analisadas pode-se observar que os vetores com maior influência sobre o eixo 1 (componente principal 1) foram aroma e sabor torrados positivamente e aroma doce e sabor ácido negativamente. Com relação ao eixo 2 (componente principal 2) a maior influência foi alcançada pelos atributos aroma ácido e cor marrom positivamente e sabor e aroma chocolate negativamente. Atributos com menor influência sobre os dois eixos foram adstringência, aparência gordurosa, aparência encorpada e sabor amargo. 
Segundo a Figura 2 a amostra de cacau torrada ocupou região bem distinta no gráfico, diferenciando-se bastante das amostras de cupuaçu. As amostras de cupuaçu torradas por 38 e 40 minutos apresentaram-se mais próximas entre si e pouco distantes das amostras torradas por 42 e 44 minutos, que por sua vez ocuparam regiões próximas entre si, estando a amostra 42 levemente deslocada em direção à amostra de cacau. A amostra de cacau torrada por 38 minutos caracterizou-se, principalmente, pela intensidade maior de sabor e aroma torrados, aparência gordurosa, aparência encorpada, sabor amargo e sabor chocolate. As amostras de cupuaçu torradas por 38 e 40 minutos, que visualmente são semelhantes, caracterizaram-se mais pelos sabor e aroma ácidos. No caso da amostra de cupuaçu torrada por 42 minutos observou-se maior predominância de aroma doce, sabor e aroma de chocolate, aroma e sabor levemente torrado e adstringência. Os atributos que mais caracterizaram a amostra de cupuaçu torrada por 44 minutos foram aroma e sabor de chocolate e aroma doce.

\section{FIGURA 1 - PROJEÇÃO DOS ATRIBUTOS SENSORIAS E DAS AMOSTRAS NOS COMPONENTES PRINCIPAIS I E II (ACP)}

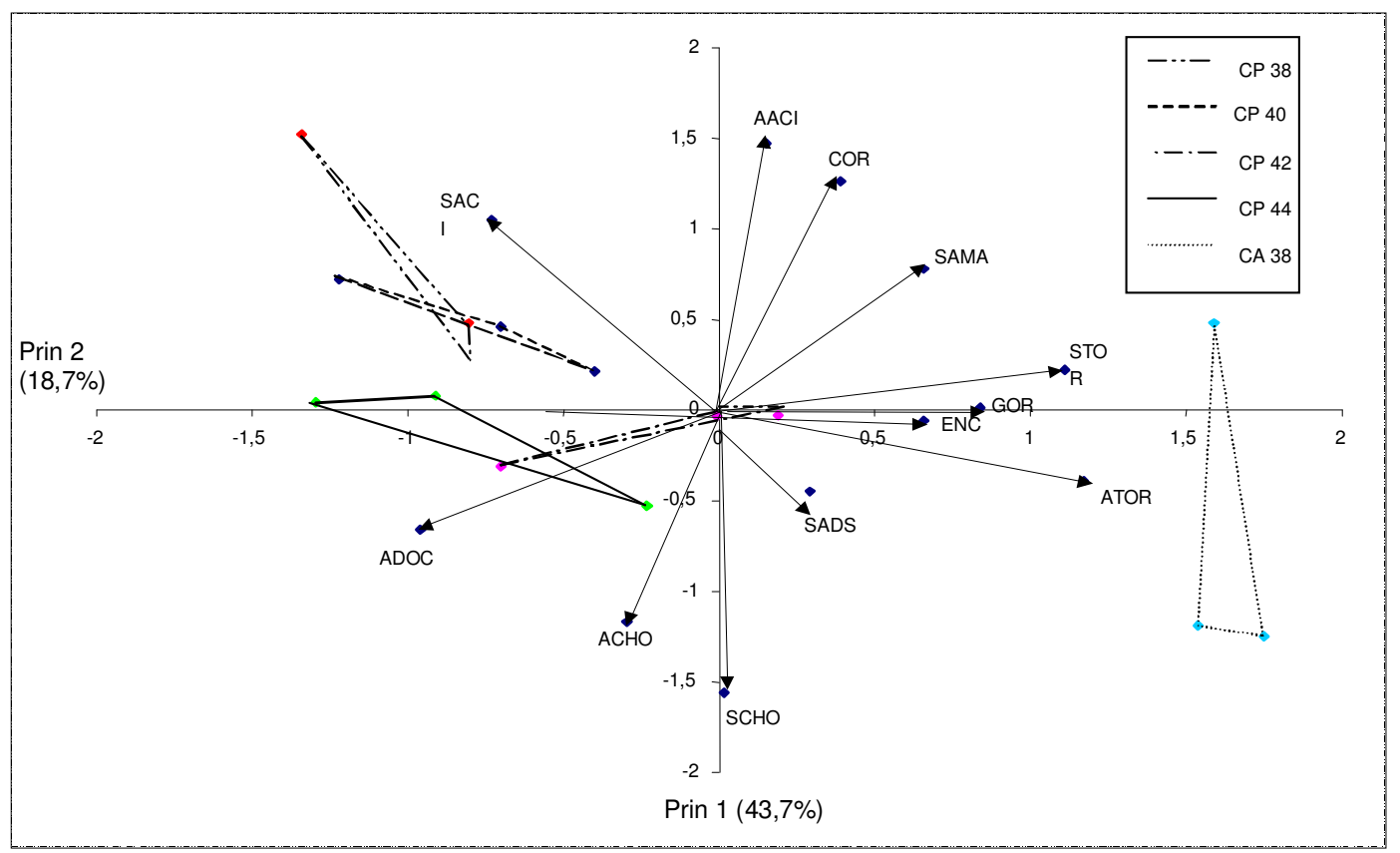

Obs.:

- vetores com medidas mais distantes de zero correspondem às variáveis com maior influência sobre o componente principal e os mais próximos à variável com menor influência;

- cada amostra é representada por um triângulo, sendo que cada vértice é definido pelas repetições apresentadas pela equipe sensorial; e

- amostras similares ocupam regiões próximas no gráfico e são caracterizadas pelos vetores (atributos) que se apresentam mais próximos a elas. 
Analisando-se mais especificamente os atributos com maior predominância, segundo a ACP (ATOR, STOR, SACI, AACI, ADOC, COR, SCHO E ACHO), pode-se observar que a amostra de cacau diferenciou-se em vários deles quando comparada às amostras de cupuaçu. A intensidade em vários destes foi muito superior para o cacau, como no caso de cor marrom, sabor torrado e aroma chocolate. Para os atributos sabor chocolate, aroma torrado, a diferença não foi tão acentuada. Já para os atributos aroma ácido e sabor ácido o cacau apresentou bastante semelhança com às amostras de cupuaçu. Observou-se ainda que a amostra de cacau caracterizou-se pela baixa intensidade de aroma doce (Figura 2).

\section{FIGURA 2 - GRÁFICO ARANHA REPRESENTANDO AS MÉDIAS DOS ATRIBUTOS PARA AS AMOSTRAS ANALISADAS}

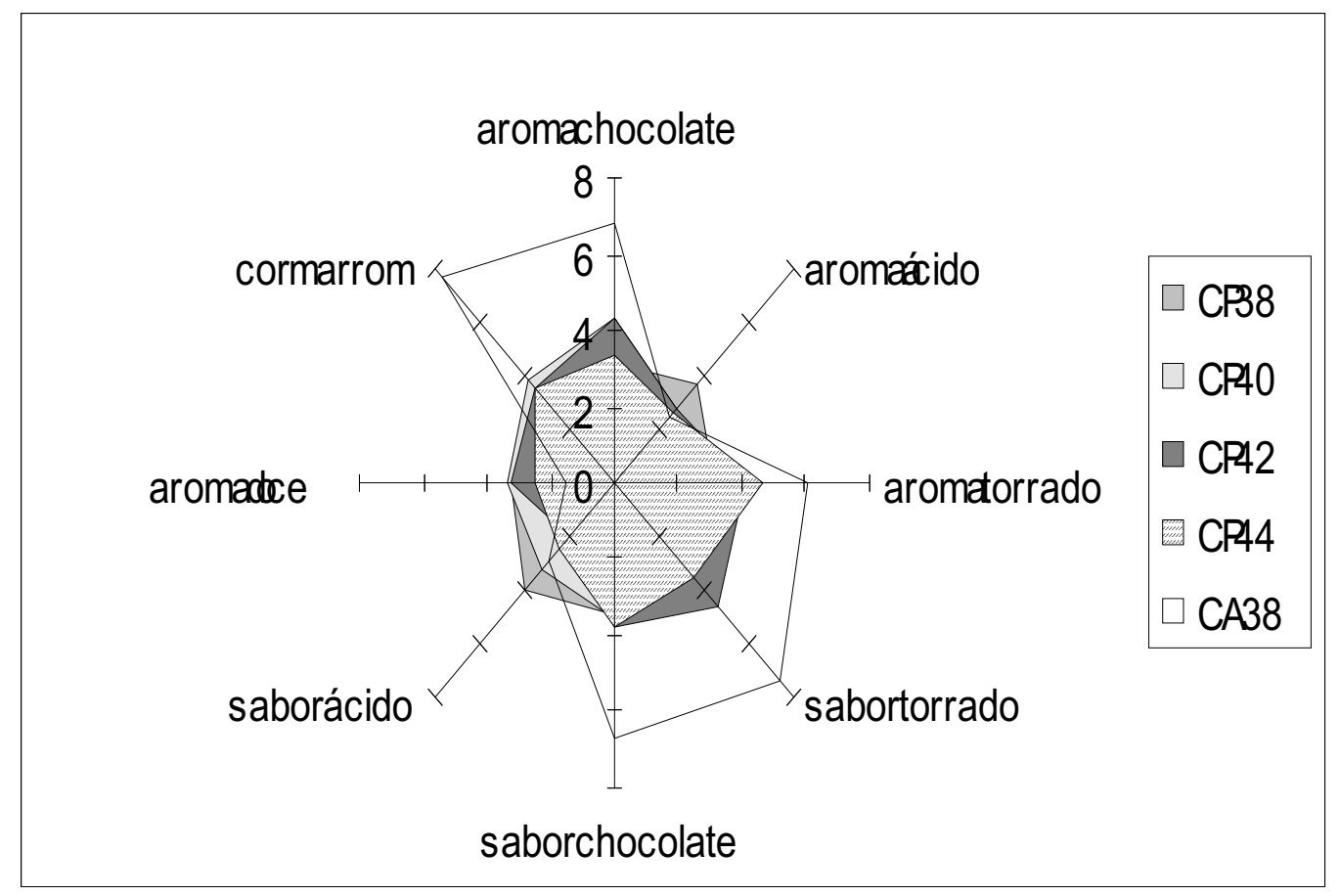

Em termos gerais, as amostras de cupuaçu torradas nos diferentes tempos apresentaram semelhança quanto aos atributos avaliados, porém a amostra 38 evidenciou maior intensidade de sabor e aroma ácido e menor sabor torrado. A amostra 40 destacou-se levemente pelo aroma de chocolate, sabor e aroma de torrado. Para a amostra 42 os resultados foram similares à amostra 40 , com menor intensidade de sabor ácido e maior intensidade de sabor e aroma torrados. A amostra de cupuaçu 
torrada por 44 minutos apresentou maior intensidade de aroma torrado e cor marrom e menor intensidade de aroma ácido, aroma doce e sabor torrado, em comparação com as demais amostras de cupuaçu. Para o atributo sabor chocolate, as amostras de cupuaçu apresentaram-se similares. Apesar de não haver diferença significativa entre elas para $\mathrm{p} \leq 0,05$, a amostra torrada por 42 minutos foi a que obteve maior média, segundo os provadores, fazendo com que fosse a escolhida para o teste de aceitação.

\subsection{TESTE DE ACEITAÇÃO}

O produto similar ao chocolate, obtido com o cupuaçu e submetido ao teste de aceitação, usando-se equipe de 40 provadores não treinados, apresentou boas características de sabor (Figura 3), sendo que $57 \%$ dos julgadores atribuíram nota 7 (gostei regularmente) para o produto.

\section{FIGURA 3 - HISTOGRAMA REFERENTE AOS RESULTADOS DO TESTE DE ACEITAÇÃO}

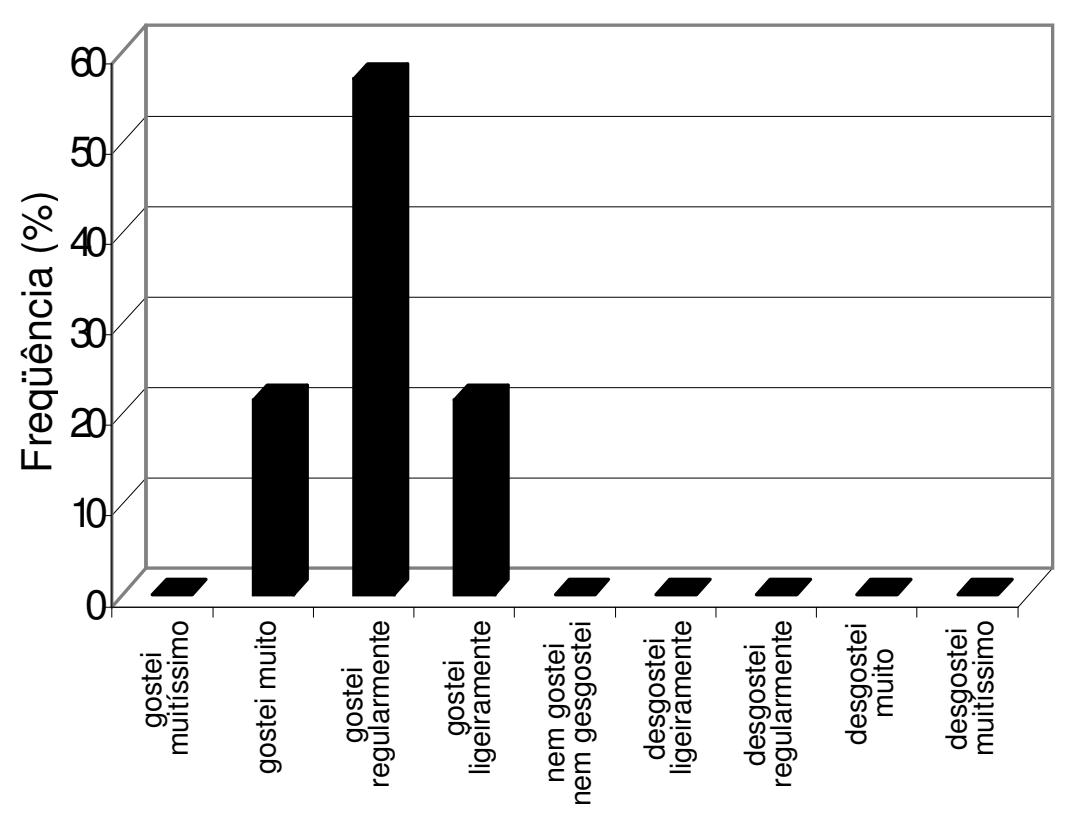


Observou-se que nenhum provador atribuiu nota menor que 6 , ficando o produto enquadrado na faixa de gostei muito e gostei ligeiramente, o que corresponde a aceitação muito boa.

Segundo os comentários anotados na ficha de avaliação, muitos julgadores indicaram que 0 produto apresentou sabor semelhante à chocolate hidrogenado, com notas mais frutais.

\title{
4 CONCLUSÃO
}

De forma geral, pôde-se observar que o aumento no tempo de torração aumentou a intensidade de cor marrom e de aroma torrado para as amostras de cupuaçu, gerando diminuição do aroma e do sabor ácido.

O cacau apresentou características sensoriais diferentes do cupuaçu, sendo bem mais ácido e amargo, com coloração mais escura e acentuado sabor de chocolate. O cupuaçu apresentou suave sabor de chocolate e sabor mais adocicado que o cacau, evidenciando sua potencialidade como matéria-prima com características peculiares de sabor, que poderiam contribuir para a obtenção de produtos diferenciados na linha dos achocolatados.

Apesar da equipe sensorial treinada ter detectado sabor e aroma de chocolate nas amostras de cupuaçu, esta amêndoa diferiu em termos gerais do sabor do cacau, apresentando características próprias e marcantes. Estas poderiam ser valorizadas na utilização do cupuaçu em misturas com o cacau para a elaboração de produtos diferenciados, como é o caso por exemplo da "gianduia" que mistura cacau com avelãs para produzir massa utilizada tanto como recheio de bombons como para a produção de chocolates.

\begin{abstract}
Descriptive terminology and sensory profile of cupuasu and cocoa roasted bean were developed by sensory descriptive analysis (QDA). Four cupuasu samples roasted in an electrical rotating oven at $150 \stackrel{\circ}{C}$ and time of $38,40,42$ and 44 minutes and one cocoa sample roasted for 38 minutes were sensory evaluated and compared. Twelve terms which best described similarities and differences among the samples were generated, being noted eight terms with greater predominance, according to the Principal Component Analysis (PCA). Nine panelists were selected on the basis of their discriminative ability $\left(F_{\text {sample }}\right.$ significant for $\left.p \leq 0.50\right)$, reproducibility of judgments $\left(F_{\text {reproducibility }}\right.$ significant for $\left.>0.05\right)$ and panel agreement. The intensity of each descriptor or attribute were fixed in an $10 \mathrm{~cm}$ unstructured scale, anchored in the ends on the terms "weak" and "strong", "low" and "high", etc. Data were analyzed by ANOVA, Tukey test and PCA, presenting results in tables and charts. It was observed that roasted cocoa presented different sensory characteristics, being sourer, bitter, darker and with accentuated chocolate taste than
\end{abstract}


cupuasu. In the other hand the cupuasu presented smoother chocolate taste and sweeter than cocoa.

\section{REFERÊNCIAS}

1 AMERINE, M. A.; PANGBORN, R.R.; ROESSLER, E.B. Principles of sensory evaluation of food. New York: Academic Press, 1965. p. 1-22.

2 ARAGÃO, C. G. Mudanças físicas e químicas da semente de cupuaçu (Theobroma grandiflorum Schum) durante o processo fermentativo. Manaus, 1992. 115 p. Dissertação (Mestrado), INPA, Universidade do Amazonas.

3 CAVALCANTE, P. B. Frutas comestíveis da Amazônia. 3. ed. Manaus: INPA, 1976. $176 \mathrm{p}$.

4 COUTINHO, R. B. S. Industrialização das sementes de cupuaçu. Revista de Farmácia e Bioquímica da Amazônia, Belém, v. 2, n. 4, p. 7-10, 1969.

5 MOSKOWITZ, H. R. Product testing and sensory evaluation of foods: marketing and R\&D approaches. West Port: Food \& Nutrition, 1983. $605 \mathrm{p}$.

6 NAZARÉ, R.F.R. Processos agroindustriais para o desenvolvimento de produtos de cupuaçu (Theobroma grandiflorum). In: SEMINÁRIO INTERNATIONAL SOBRE PIMENTA-DO-REINO E CUPUAÇU, 1., Belém, 1996. Anais... Belém, 1996. $8 \mathrm{p}$.

7 RIBEIRO, G.D. Situação atual e perspectivas da cultura do cupuaçuzeiro (Theobroma grandiflorum, Schum) no Estado de Rondônia, Brasil. In: SEMINÁRIO INTERNATIONAL SOBRE PIMENTA-DO-REINO E CUPUAÇU, 1., Belém, 1996. Anais... Belém, 1996. $7 \mathrm{p}$.

8 SAS INSTITUTE. Statistics analyses systems (SAS). Cary, USA, 1989. Version 6.

9 SILVA, M.A.A.P. Flavor properties and stability of a corn-based snack: aroma profiles by gas chromatography (GC). GC-olfactometry, mass spectometry and descriptive sensory analysis. Oregon, 1992. Tese (Doutorado), Oregon State University.

10 STONE, H.; SIDEL, J. L. Sensory evaluation practices. Orlando, USA: Academic Press, 1985. p. 296. 
11 VENTURIERI, G. A.; AGUIAR, J. P. L. Composição do chocolate caseiro de amêndoas de cupuaçu (Theobromna grandiflorum Schum). Acta Amazônica, Manaus, v. 18, n. 1/2, p. 3-8, 1988. 